\title{
Brief Therapy for Post-Traumatic Stress Disorder Symptoms in Maltreated Youth in a Shelter Care Facility
}

\author{
Lisa M. Linning ${ }^{\#}$ and Christopher A. Kearney ${ }^{\#^{*}}$
}

University of Nevada, Las Vegas, Department of Psychology, 4505 Maryland Parkway, Las Vegas, NV 89154-5030, USA

\begin{abstract}
Therapy to address post-traumatic stress disorder (PTSD) symptoms in maltreated youth has received increased research attention in recent years. However, little data are available regarding the treatment of youth temporarily housed in shelter care facilities when parents are unavailable or inaccessible. The present study examined a brief group therapy protocol for such youth in a county-operated shelter. Participants received 1-6 sessions of treatment depending on their length of stay at the shelter. Treatment components included psychoeducation, anxiety management, cognitive restructuring and coping skills, emotional expression, mindfulness, exposure-based practice, boundary setting, and journaling. Results revealed a significant reduction in PTSD symptoms overall and the number of group therapy sessions mediated pre-treatment and post-treatment scores on various dependent measures. In addition, level of pretreatment dissociation predicted reduction in PTSD levels. These preliminary results indicate that brief treatment for youth temporarily housed in a shelter care facility can provide some amelioration of PTSDrelated symptoms.
\end{abstract}

Keywords: Maltreatment, Post-traumatic stress disorder, Youth, Group therapy, Temporary shelter.

\section{INTRODUCTION}

Trauma-focused therapy for maltreated children and adolescents has gained increased research attention in recent years. Such therapy has typically focused on symptoms of post-traumatic stress disorder (PTSD) in maltreated youth, which are often varied and substantial. PTSD rates among maltreated children range from $11.1 \%$ to $70.8 \%[1,2]$. Maltreated youth in out-of-home care settings often display post-traumatic symptoms as well $(19.2 \%)$ [3]. In particular, PTSD rates are considerable among those victimized by sexual maltreatment $(21-50 \% ; 42-90 \%$ in clinical samples), physical maltreatment $(50 \%)$, or neglect with domestic violence (33-50\%) [4].

Trauma-focused therapy generally involves cognitive and behavioral components to address emotional regulation and coping skills and includes various forms of exposure-based practice. Trauma-focused cognitive-behavioral therapy (TFCBT) has been developed to treat symptoms of post-traumatic stress disorder in youth who have been sexually maltreated. TF-CBT involves

Address corresponding to Christopher A. Kearney, University of Nevada, Las Vegas, Department of Psychology, 4505 Maryland Parkway, Las Vegas, NV 89154-5030, USA; Tel: 702-895-3305; Fax: 702-895-0195. E-mail: chris.kearney@unlv.edu

\#Authors contributed equally to this work. psychoeducation, parenting skills, relaxation, emotional regulation, cognitive coping, trauma narratives, exposure-based practices, and safety enhancement [5].

TF-CBT was found to be effective in several studies [6]. The therapy is also effective for 3-6-yearold children and for youth presenting with continuous traumas $[7,8]$. In addition, reviews of treatment for PTSD in children generally involve treatment for PTSD symptoms in maltreated youth [9].

Deblinger and colleagues stated that alternative formats of TF-CBT may be effective but remain largely untested [5]. Such testing, however, is important because many clinicians who utilize the therapy typically employ only several and not all of the strategies [10]. In addition, cognitivebehavioral approaches for maltreated youth with PTSD symptoms often include parents, but parents are often unavailable or inaccessible when youth are placed in shelter care [11]. Evaluations of forms of TF-CBT specifically for sheltered youth are needed.

Cohen and colleagues stated that TF-CBT has been evaluated in research settings as well as community mental health centers and foster care placements [12,13]. However, little information is available as to whether components of this 
approach might be useful or effective for maltreated youth removed from their homes and placed in a county-operated child protection shelter. A key purpose of the current study was to examine components of TF-CBT in a limited format for sheltered youth who were available for intervention for various and brief periods. In addition, treatment was targeted toward youth only, because parents were unavailable or inaccessible.

We hypothesized that brief therapy for traumatized children would produce significant reductions in PTSD symptoms. In addition, the number of group sessions was expected to mediate the relationship between pre-treatment and posttreatment PTSD symptoms. Problem-solving and seeking social support scores were also expected to be significantly different between pre-treatment and post-treatment.

\section{METHOD}

Participants were recruited from a countyoperated child protection shelter that provides respite care to maltreated youth. Participants $(n=$ 54) were aged $12-17$ years and were multiracial (24.1\%), European American (24.1\%), AfricanAmerican (21.4\%), Hispanic (19.0\%), Asian $(6.9 \%)$, or other $(1.4 \%)$. Most $(63.8 \%)$ were female and many came from single-parent homes $(44.8 \%)$, lived more than 3 months with a relative or in state custody (43.1\%), had a family member who had been imprisoned $(79.7 \%)$, and reported regular drug/alcohol use in their home (47.5\%). Youth with significant cognitive impairment (e.g., documented intellectual disability) or behavior problems (e.g., inability to manage anger or physical aggression) were excluded. Youth who received psychotropic medication or who failed to attend therapy sessions or complete all pre- and post-assessment measures were also excluded.

Four youth assessment measures were employed. When Bad Things Happen (WBTH) is a 90-item self-report measure that assesses PTSD diagnostic and associated trauma-related symptoms in children and adolescents [14]. Associated symptoms include anxiety, depression, omens, survivor guilt, self-blame, fantasy denial, selfdestructive thoughts and behaviors, dissociation, antisocial behavior, risk-taking behaviors, and changes in eating habits. The WBTH yields two scores for total PTSD symptoms endorsed by the child as well as a PTSD severity score. The measure has good internal consistency (Cronbach's alpha, .92) as well as convergent validity.

Coping Strategy Inventory (CSI) is a 33-item selfreport measure that assesses how youth cope with problems in their lives [15]. Respondents describe a recent (past 6 months) stressful event that required them to make an important decision and then endorse specific coping behaviors used to manage the stressful decision. Responses are summed into 3 subscales: problem solving, seeking social support, and avoidance. The CSI has good internal consistency (mean alpha coefficient, .89). The scale has also demonstrated concurrent validity [15].

A Satisfaction Survey was provided to participants when they departed the group that included a list of therapy components and participants were asked to endorse what they liked most and least about the therapy sessions. Participants could also add their own comments. Each participant was also asked if therapy was helpful.

The Children's PTSD Inventory (CPTSD-I), a clinician assessment was also conducted [16]. The CPTSD-I is a semi-structured interview to assess PTSD symptoms for primary and secondary traumatic events in youth aged 7-18 years. The CPTSD-I assesses exposure to trauma and situational reactivity, re-experiencing symptoms, avoidance and numbing symptoms, increased arousal, and significant distress. The CPTSD-I also assesses duration of distress for each symptom group. Total symptoms were used for this study. The CPTSD-I also requires youth to identify their most traumatizing event. The CPTSD-I has good internal consistency (Cronbach's $\alpha$ : .53-.89). The CPTSD-I subscales also have good interrater (Cohen's K: .84-1.00) and test-retest (Cohen's K: .78-1.00) reliability. The CPTSD-I has displayed strong concurrent, convergent, and discriminant validity.

Procedures and measures were approved by a university-based institutional review board and a county district attorney. Permission to interview and informed consent were obtained from the di- 
rector of the county-operated shelter. No parent or other collateral data were available due to administrative constraints. Assent from each participant was obtained prior to data collection. Participants were assessed and treated at the shelter. Participants were at the shelter for varying periods of time while they awaited foster care or adoption placements. Participants ended treatment when they left the shelter; participants attended $1(n=$ $7), 2(n=5), 3(n=5), 4(n=10), 5(n=8)$, or $6(n$ $=19)$ sessions $(M=3.9, S D=2.0)$.

Therapy was provided in 90-minute consecutive weekly sessions, facilitated by the primary investigator and attended by two silent co-therapists. The co-therapists provided additional adult supervision to ensure protocol adherence. Participants were reminded at the start of group therapy that other members would hear information they disclosed during sessions, so participants were given an opportunity to discuss sensitive material privately with the primary investigator if desired. Long-term follow-up was not possible due to each youth's confidential placement.

Participants were assessed when present and available and when researchers were at the shelter. A verbal description of the study was presented to all youth in the residential cottages. Those who volunteered to participate were assessed for the study. Participants with PTSD symptoms were invited to engage in up to 6 weekly group therapy sessions.

The intervention (Brief Therapy for Traumatized Children, or BTTC) was designed to teach youth essential coping and anxiety management skills in a time-limited format to help them recover from traumatic events. Sessions were conducted in a group therapy format to maximize service delivery and to provide peer support. Treatment components consisted of psychoeducation, anxiety management, cognitive restructuring and coping skills, emotional expression, mindfulness, exposure-based practice, boundary setting, and journaling. These components are briefly described here.

Psychoeducation involved providing a conceptual and explanatory framework for youth to understand anxiety and related symptoms experienced since the traumatic event. The concept of traumatic stress was reviewed and the therapy process explained to help youth understand the nature and origin of their symptoms, to normalize reactions, and to provide a rationale for the therapy components. Anxiety management techniques involved helping youth develop skills for selfsoothing and arousal reduction. Participants learned to identify anxiety symptoms and used deep diaphragmatic breathing, progressive muscle relaxation, and visual imagery to manage the symptoms.

Cognitive restructuring involved identifying distortions related to self-blame, catastrophizing, exaggerating the importance of negative events, or minimizing personal accomplishments and selfworth. Youth were taught to replace distortions with more adaptive internal verbalizations. Emotional expression involved teaching youth to develop a vocabulary for emotions, practice expressing emotions felt currently and when recalling traumatic events, and recognize cues from others to identify their emotional states. Cognitive restructuring was integrated with cognitive coping skills training to help youth manage emotions such as anger when discussing traumatic events.

Mindfulness involved helping youth become more aware of current emotions and bodily sensations and to accept them. This was designed to reduce dissociation, boost present-awareness, and prepare youth for exposure-based practice. Exposurebased practice involved opportunities for youth to practice controlling their anxiety and related symptoms while recalling traumatic events. The procedure initially involved imaginal exposure (e.g., the therapist related an imaginary anxiety-provoking story) and later involved group members who conveyed their own traumatic experiences while practicing other skills.

Boundary setting involved establishing personal boundaries to help participants develop more effective coping strategies with others, such as being assertive, setting limits, and practicing selfprotective habits. Journaling was given as homework between sessions to help youth identify and monitor emotions, thoughts, and behaviors they had concerning traumatic events. 
Structural equation modeling was used in analyzing the collected data because it provided overall goodness-of-fit estimates, allowed analysis of multiple subscales or factors, and minimized measurement error [17]. Structural equation modeling gave information about the impact directly from one variable to another, as well as the impact of mediating variables. Three models were evaluated. The models examined whether number of group sessions influenced treatment outcome as measured by CPTSD-I, WBTH, and CSI symptom scores from pre-treatment to post-treatment. Goodness-of-fit indices to test these models included the comparative fit index (CFI), Bollen's incremental fit index (IFI), and standardized root mean square residuals (SRMR). Acceptable goodness-of-fit criteria were defined as CFI and IFI values of .90+ and SRMR values of <.10 [18].

If a model had good fit, then multistep mediational analysis was conducted using structural equation modeling [19]. The predictor-outcome path $(A \rightarrow C$; pre-treatment and post-treatment scores) was first examined for adequate fit. If fit was adequate, the predictor-mediator-outcome path (mediator, number of treatment sessions) was examined. If fit occurred, the $A \rightarrow B \rightarrow C$ path was examined further under two conditions. Condition one was when the $A \rightarrow C$ path was constrained to zero; condition two was when the $A \rightarrow C$ path was not constrained to zero. Mediation occurred if the unconstrained model did not provide better fit than the constrained model.

\section{RESULTS}

A chi-squared test revealed no significant differences with respect to response to treatment as a function of gender, race, parental status, physical or sexual maltreatment, or witnessing domestic violence. The first model examined whether the number of group sessions attended influenced treatment outcome as measured by CPTSD-I symptom change scores from pre-treatment to post-treatment. Adequate fit was identified for the $\mathrm{A} \rightarrow \mathrm{C}$ model $(\mathrm{CFI}=.93, \mathrm{IFI}=.94, \mathrm{SRMR}=.08)$ and the $\mathrm{A} \rightarrow \mathrm{B} \rightarrow \mathrm{C}$ model $(\mathrm{CFI}=.93, \mathrm{IFI}=.94$, SRMR $=.08)$. Additionally, the constrained $\mathrm{A} \rightarrow \mathrm{B} \rightarrow \mathrm{C}$ model demonstrated adequate fit (CFI = $.93, \mathrm{IFI}=.93, \mathrm{SRMR}=.10)$ and was not signifi- cantly different than the unconstrained model. Number of group sessions thus mediated the relationship between CPTSD-I pre-treatment and post-treatment scores. Total PTSD symptoms post-treatment $(M=16.74, S D=10.38)$ were significantly lower than total PTSD symptoms pretreatment $(M=21.29, S D=10.16)(t(57)=6.39$, $\mathrm{p}<.001)$.

The second model examined whether the number of group sessions attended influenced treatment outcome as measured by WBTH symptom change scores from pre-treatment to posttreatment. This model did not meet criteria for mediation. However, WBTH PTSD symptoms post-treatment $(M=39.87, S D=19.50)$ were significantly lower than WBTH PTSD symptoms pretreatment $(M=49.36, S D=23.86)(t(57)=4.39$, $p<.001)$. The third model examined whether the number of group sessions attended influenced treatment outcome as measured by CSI problemsolving and seeking social support change scores from pre-treatment to post-treatment. Adequate fit was identified for the $A \rightarrow C$ model $(C F I=.93, I F I=$ .93$, SRMR $=.06)$ and the $A \rightarrow B \rightarrow C$ model $(C F I=$ $.93, \mathrm{IFI}=.94, \mathrm{SRMR}=.05)$. Additionally, the constrained $\mathrm{A} \rightarrow \mathrm{B} \rightarrow \mathrm{C}$ model demonstrated adequate fit $(\mathrm{CFI}=.93$, IFI $=.94, \mathrm{SRMR}=.05)$ and was not significantly different from the unconstrained model. The number of group sessions attended mediated the relationship between CSI pretreatment and post-treatment scores.

Post hoc analyses were conducted. Stepwise regression regarding CPTSD-I and CSI symptom change scores revealed no significant predictors of change. However, pre-treatment dissociation predicted change in WBTH PTSD scores ( $F(1$, $52)=5.32, p<.05)$, accounting for $9.1 \%$ of the variance. Satisfaction results revealed that participants most liked discussing their emotions $(31.4 \%)$ and learning new skills (20.4\%). Participants endorsed the amount of paperwork (14.8\%) and personal questions $(13.0 \%)$ as what they liked least $(55.6 \%$ left this blank or specifically wrote the word "nothing"). Participants endorsed the treatment as helpful $(n=40)$ or not helpful $(n=$ 5). 


\section{DISCUSSION}

This study demonstrated on a preliminary basis the effectiveness of a brief trauma-focused group treatment therapy for maltreated youth in shelter care. Literature is sparse regarding the treatment of youth with post-traumatic symptoms in shelter care who have been removed from their homes due to maltreatment. Results of this study revealed that such youth may benefit from group treatment, particularly when they are able to attend up to 6 weekly sessions. A negative developmental trajectory is common in this population, so identifying ways to ameliorate post-traumatic symptoms within shelter care settings could also be an important approach for tertiary prevention.

Research concerning traumatized youth indicates that a supportive therapeutic environment and an opportunity to verbally process traumatic events are positively related to PTSD symptom reduction $[20,21,22]$. Group therapy provides an opportunity for victims to join with others who may feel isolated and alienated by traumatic experiences. Interactions with group members within a safe therapeutic environment can provide validation, support, connectedness, and strength to help members manage the challenging task of processing traumatic material [23]. In addition, length of treatment relates positively to PTSD symptom reduction $[24,25]$.

Another key finding of the study was that pretreatment dissociation levels predicted symptom change on WBTH PTSD scores. Participants with high pre-treatment dissociation reported significant symptom reduction post-treatment. These data are encouraging because key treatment components, particularly proactive coping strategies, were designed to address this symptom. Dissociation may be a predictor of PTSD development, increased PTSD symptom severity, and higher levels of distress, though this remains controversial [26].

Dissociation can serve a trauma victim in several ways, including escape from reality, an analgesic effect to reduce pain, and an alteration of the sense of self. Putnam stated that the effects of dissociation could be characterized as a defense mechanism, avoidance of reality, or avoidance of the pain associated with overwhelming trauma [27]. Additionally, some researchers have postulated that emotional numbing is an element of dissociation. Emotional numbing and dissociation have a strong relationship with PTSD, and both have been characterized as negative coping strategies [28].

Another key finding of the study was that the number of group sessions attended influenced problem-solving and seeking social support change scores. Previous research has shown that cognitive avoidance after traumatic events may lead to increased risk of psychological symptoms [29]. In addition, avoidant coping may suggest an inability to emotionally engage with traumatic memory, which can interfere with processes that lead to recovery [30].

Limitations of the present study must be considered. First, assessment was based on youth selfreport given their special circumstances and administrative constraints. Second, other variables that could have affected the treatment process, such as depression and therapeutic alliance, were not explicitly measured [31]. Third, the study was uncontrolled because all youth at the shelter had experienced maltreatment. However, the number of treatment sessions as a differentiating variable was considered. Fourth, follow-up data collection was not possible, which limits our understanding of whether treatment gains were maintained. Finally, sample size was limited but was ethnically diverse, and psychometricallystrong measures and data analytic strategies were utilized.

Despite these limitations, the present study provided some evidence that adolescents who participated in brief therapy experienced a reduction in PTSD symptoms. Future controlled research is needed to further test this intervention and identify its most effective components. Further evaluation of optimal number of sessions would also be helpful. Such work could provide mental health professionals in shelter care facilities with knowledge about cost-effective and efficacious strategies to thwart post-traumatic symptoms in maltreated youth. 


\section{REFERENCES}

[1] Saigh PA, Yasik AE, Sack WH, Koplewicz HS. Child-adolescent posttraumatic stress disorder: Prevalence, risk factors and comorbidity. In: Saigh, PA, Bremner JD, editors. Posttraumatic stress disorder: A comprehensive text. Boston: Allyn and Bacon; 1999. p. 18-43.

[2] Yehuda R, Spertus IL, Golier JA. Relationship between childhood traumatic experiences and PTSD in adults. In: Eth S, editor. PTSD in children and adolescents. Washington, DC: American Psychiatric Publishing; 2001. p. 117-58.

[3] Kolko DJ, Hurlburt MS, Zhang J, Barth RP, Leslie LK, Burns BJ. Posttraumatic stress symptoms in children and adolescents referred for child welfare investigation: A national sample of in-home and out-of-home care. Chil Maltreat 2010; 15: 48-63.

[4] Kearney CA, Wechsler A, Kaur H, Lemos-Miller A. Posttraumatic stress disorder in maltreated youth: A review of contemporary research and thought. Clin Chil Fam Psychol Rev 2010; 13: 46-76.

[5] Deblinger E, Mannarino AP, Cohen JA, Runyon, MK, Steer RA. Trauma-focused cognitive behavioral therapy for children: Impact of the trauma narrative and treatment length. Dep Anx 2011; 28: 67-75.

[6] Silverman WK, Ortiz CD, Viswesvaran C, Burns BJ, Kolko DJ, Putnam FW, Amaya-Jackson L. Evidence-based psychosocial treatments for children and adolescents exposed to traumatic events. J Clin Chil Adol Psychol 2008; 37: 15683.

[7] Scheeringa MS, Weems CF, Cohen JA, AmayaJackson L, Guthrie D. Trauma-focused cognitivebehavioral therapy for posttraumatic stress disorder in three through six year old children: A randomized clinical trial. J Chil Psychol Psychiat 2011; 52: 853-60.

[8] Murray LK, Cohen JA, Mannarino AP. Traumafocused cognitive behavioral therapy for youth who experience continuous traumatic exposure. J Peace Psychol 2013; 19: 180-95.

[9] Kowalik J, Weller J, Venter J, Drachman D. Cognitive behavioral therapy for the treatment of pediatric posttraumatic stress disorder: A review and meta-analysis. J Behav Ther Experiment Psychiat 2011; 42: 405-13.

[10] Allen B, Johnson JC. Utilization and implementation of trauma-focused cognitive-behavioral therapy for the treatment of maltreated children. Chil Maltreatment 2012; 17: 80-5.

[11] Kolko DJ, Iselin A-MR, Gully KJ. Evaluation of the sustainability and clinical outcome of Alternatives for Families: A Cognitive-Behavioral Ther- apy (AF-CBT) in a child protection center. Chil Ab Negl 2011; 35: 105-16.

[12] Cohen JA, Berliner L, Mannarino A. Trauma focused CBT for children with co-occurring trauma and behavior problems. Chil Ab Negl 2010; 34: 215-24.

[13] Cohen JA, Mannarino AP, lyengar, S. Community treatment of posttraumatic stress disorder for children exposed to intimate partner violence: $A$ randomized controlled trial. Arch Ped Adol Med 2011; 165: 16-21.

[14] Fletcher KE. Childhood posttraumatic stress disorder. In: Mash EJ, Barkley RA, editors. Child psychopathology. New York: Guilford; 1996. p. 242-76.

[15] Amirkhan JH. Criterion validity of a coping measure. J Personality Assess 1994; 62: 242-61.

[16] Saigh PA, Yasik AE, Oberfield R, Green BL, Halamandaris PV, McHugh M. The Children's PTSD Inventory: Development and reliability. J Traumatic Stress 2000; 13: 369-80.

[17] Bentler PM, Wu EJC. EQS 6.1 for Windows: Multivariate software. Encino, California: Author; 2005.

[18] Kline RB. Principles and practice of structural equation modeling. 2nd ed. New York: Guilford; 2005.

[19] Holmbeck G. Toward terminological, conceptual and statistical clarity in the study of mediators and moderators: Examples from the child-clinical and pediatric psychology literatures. J Consult Clin Psychol 1997; 65: 599-610.

[20] Cohen JA, Berliner L, March JS. Treatment of children and adolescents. In Foa EB, Keane TM, Friedman MJ, editors. Effective treatments for PTSD: Practice guidelines from the International Society for Traumatic Stress Studies. New York: Guilford; 2000. p. 106-38.

[21] DeRubeis RJ, Brotman, MA, Gibbons, CJ. A conceptual and methodological analysis of the nonspecifics argument. Clin Psychol: Sci Prac 2005; 12: 174-83.

[22] Kinzie JD. Psychotherapy for massively traumatized refugees: The therapist variable. Am J Psychother 2001; 55: 475-90.

[23] Foy DW, Glynn SM, Schnurr PP, Jankowski MK, Wattenberg MS, Weiss MS, Marmar CR, Gusman FD. Group therapy. In: Foa EB, Keane $T M$, Friedman MJ, editors. Effective treatments for PTSD: Practice guidelines from the International Society for Traumatic Stress Studies. New York: Guilford; 2000. pp. 155-75.

[24] Cloitre M, Stovall-McClough KC, Miranda R, Chemtob CM. Therapeutic alliance, negative mood regulation, and treatment outcome in child 
abuse-related posttraumatic stress disorder. J Consult Clin Psychol 2004; 72: 411-6.

[25] Cohen JA, Deblinger E, Mannarino AP. Traumafocused cognitive-behavioral therapy for sexually abused children. In: Hibbs ED, Jensen, PS, editors. Psychosocial treatments for child and adolescent disorders: Empirically based strategies for clinical practice. 2nd ed. Washington, DC: American Psychological Association; 2005; p. 743-65.

[26] Bryant RA. Does dissociation further our understanding of PTSD? J Anx Dis 2007; 21: 183-91.

[27] Putnam FW. Dissociation in children and adolescents: A developmental perspective. New York: Guilford; 1997.
[28] Feeny NC, Zoellner LA, Fitzgibbons LA, Foa EB. Exploring the roles of emotional numbing, depression, and dissociation in PTSD. J Traumatic Stress 2000; 13: 489-98.

[29] Ehlers A, Mayou RA, Bryant B. Cognitive predictors of posttraumatic stress disorder in children: Results of a prospective longitudinal study. Beh Res Ther 2003; 41: 1-10.

[30] Salmon K, Bryant RA. Posttraumatic stress disorder in children: The influence of developmental factors. Clin Psychol Rev 2002; 22: 163-88.

[31] Linning LM, Kearney CA. Posttraumatic stress disorder in maltreated youth: A study of diagnostic comorbidity and child factors. J Interpers Viol 2004; 19: 1087-101. 